\section{A developmental approach to induced pluripotent stem cells-based tissue engineered heart valves}
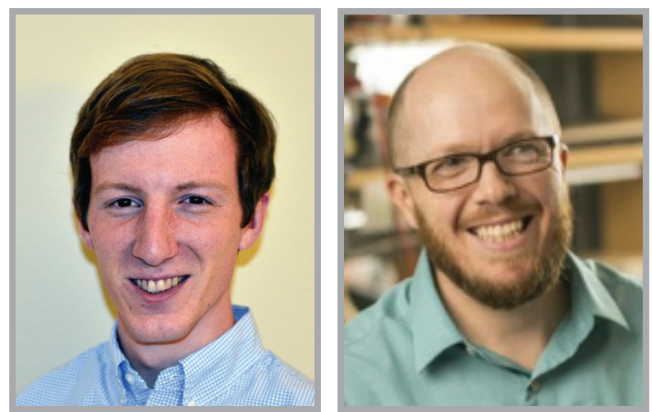

\author{
“...it is clear that a novel \\ approach to generate \\ engineered valve interstitial \\ cells is needed, and induced \\ pluripotent stem cells could \\ be used to meet this need."
}

Mark J Vander Roest ${ }^{1} \&$ W David Merryman*,1

First draft submitted: 21 October 2016; Accepted for publication: 26 October 2016; Published online: 19 December 2016
Valvular heart disease constitutes a large fraction of cardiac disease in both pediatric and adult patients. Several pharmacological strategies are under investigation to treat age-related valve pathologies such as calcification, but none have produced clinically viable targets to reverse or even stop disease progression [1]. Additionally, developmental abnormalities in valve formation cannot typically be addressed by pharmacological intervention. In both cases, total viable treatment option.

Cardiac valve replacement is becoming an increasingly prevalent procedure. Over 280,000 total valve replacements are performed annually worldwide [2], with the two primary causes being congenital valve malformation and age-related degeneration. Currently, the two available options for replacement valves are tissue-based bioprosthetics or mechanical valves, both of which have serious drawbacks. Bioprosthetic valves, which are typically made from fixed porcine valve replacement is commonly the only valves or bovine pericardium, are prone to long-term mechanical complications, including valvular stiffening as a result of fibrosis and calcification or tissue degradation [3]. Mechanical replacements are more durable, but major failures have occurred in certain models in the past [4], and recipients require anticoagulant treatment for life. Traditionally, implantation has required open chest surgery, but transcatheter aortic valve replacement is now an accepted procedure for bioprosthetic valve implantation that has shown reduced morbidity [5]. Nevertheless, mechanical failure of tissue-based valves limits their practicality in younger patient populations, as multiple replacements may be necessary. Furthermore, neither type of valve replacement is capable of long-term growth and remodeling, meaning pediatric recipients require subsequent replacements throughout childhood.

Given the shortcomings of current valve replacement options, tissue-engineered heart valves (TEHVs) have been proposed

\section{KEYWORDS}

- heart valve disease $\bullet$ induced pluripotent stem cells

- tissue-engineered heart valves

"Currently, the two available options for replacement valves are tissue-based bioprosthetics or mechanical valves, both of which have serious drawbacks."
'Department of Biomedical Engineering, Vanderbilt University, Nashville, TN 37232, USA

*Author for correspondence: Tel.: +1 615322 7219; Fax: +1 615322 6541; david.merryman@vanderbilt.edu 


\section{"Current strategies to create a novel \\ differentiation scheme focus on cytokine and small molecule inhibitor treatment to drive differentiation, and many existing principles could certainly be applied to the development of a valve endocardial cell to valve interstitial cell differentiation strategy."}

as an alternative that could combine both the hemodynamic benefits of a tissue-based valve with the longevity of a mechanical valve, while providing growth potential for pediatric patients. In principle, a TEHV can be constructed from the combination of an appropriate biomaterial and cell source, and several options for both components have been studied in various combinations [6-8]. Unfortunately, no construct researched to date has produced a TEHV viable for long-term implantation, with the major challenge being identification of a cell source that does not promote either degradation or fibrosis of the construct. In the mature valve, valve interstitial cells (VICs) constitute the majority of the cell population, with valve endocardial cells (VECs) lining the valve surface. VICs are responsible for maintenance of the extracellular matrix of the valve body and are thus the main cell type desired for development of a TEHV. Unfortunately, no cell source has been found to be capable of replicating the VICs' unique phenotype, pointing to the need for a new approach.

\section{Induced pluripotent stem cells as a potential cell source for TEHVs}

The use of patient-derived induced pluripotent stem cells (iPSCs) driven down a similar developmental pathway as VICs is one potential method for generating a suitable cell line for populating a TEHV. Human iPSCs were first generated roughly a decade ago by reprogramming fully differentiated fibroblasts via introduction of four factors [9]. Since this Nobel Prize winning discovery, iPSCs have been heralded as a means to create any tissue type from an inexhaustible supply of donor cells. Their pluripotency enables them to be differentiated to any cell type (excluding extra-embryonic cells), and generating patient-specific iPSCs from donor cells such as dermal fibroblasts, is a relatively straightforward process that ensures genetic similarity to the recipient, thereby lowering the risk of immune rejection following transplant [10].

Although no protocol to generate VICs from a pluripotent stem cell source yet exists, there is a reason to believe such a method could be created. In addition to many others, similar differentiation protocols have been established for fibroblasts, vascular smooth muscle cells and cardiomyocytes. Perhaps more relevant to VIC development, however, are the various methods for generating endothelial cells. During embryonic development, valves form when a subset of VECs overlying the endocardial cushions in the heart tube undergo endothelial to mesenchymal transformation (EMT) and invade the cushions to become VICs. The endocardial origin and EMT process which give rise to VICs may be fundamental to the heterogeneous phenotype of VICs and are certainly crucial components of using in vitro cell culture to replicate embryonic development of this cell line.

Current strategies to create a novel differentiation scheme focus on cytokine and small molecule inhibitor treatment to drive differentiation, and many existing principles could certainly be applied to the development of a VEC to VIC differentiation strategy. Several protocols have outlined methods for the generation of vascular endothelial cells, a lineage similar to endocardial cells. These methods generally use a stage of mesoderm induction via Wnt activation followed by endothelial specification through vascular endothelial growth factor treatment and purification of the resultant endothelial cells. Optimizing existing protocols for the development of endocardial specific cells could be done via the addition of growth factors known to be secreted from the myocardium, which develops in close proximity to the endocardium. Furthermore, endocardial EMT is known to be regulated by a host of paracrine signaling molecules secreted by neighboring myocardial and endocardial cells. In practice, creating a novel differentiation strategy by modulating the soluble signaling environment is the most straightforward approach, but this alone may not be sufficient to specify a VEC and subsequent VIC lineage [11].

\section{Mimicking valvular developmental mechanobiology as in vitro stimulus}

Due to the nature of its function, the heart is a mechanically complex environment, with dynamic mechanical stimuli simultaneously delivered to local tissues. Among these are mechanical strain imparted by active contraction of cardiomyocytes and hemodynamic shear stress delivered to the endocardium from the flow of blood. Both of these are known regulators of cell function and more recently have been demonstrated as having significant effects on the differentiation and maturation of other iPSC-derived cell types. The exact method in which these stimuli direct cell differentiation is the subject of ongoing research within the field 
of biomechanics, but early results may already be drawn upon to aid in development of a scheme to create a TEHV cell source $[12,13]$.

Hemodynamic shear stress refers to the frictional force imparted by the blood on the endothelial layer lining the heart and vasculature. Shear stress on the developing valve is especially dynamic, owing to the high volume of blood flow across the surface of the endocardial cushions as well as the unique cushion structure as compared with the rest of the vasculature [12]. Fluid shear has been shown in both murine embryonic endothelial cells and porcine aortic VECs to be a positive regulator of EMT [14,15], further suggesting a role for shear in driving endocardial EMT during development. Incorporating fluid shear stress into cell culture systems adds a significant amount of complexity to the cell culture process, but can be achieved with a number of commercial bioreactor systems or a setup of custom design. Optimizing shear stress profiles to match in vivo conditions and promote endocardial development and EMT will likely entail a trial and error approach to produce any definite conditions, but can be informed by computational studies of the effects of hemodynamic patterns on valve development $[13,16]$.

Cardiac contraction is another major source of mechanical stimulus to the developing valve. Strain within the heart is registered by mechanosensitive proteins such as cadherins and integrins, which transduce the mechanical signal to result in transcriptional and phenotypic changes within the cell $[17]$. Strain has been shown to increase EMT of endocardial cells in avian endocardial cushion explants and is implicated in remodeling of the developing valve by newly transformed VICs [18,19]. In a similar way, iPSC-derived cardiomyocytes have recently been shown to exhibit increased maturity in response to stress conditioning of cardiac tissue constructs [20]. In vitro reaplication of this mechanical stimulus is challenging, but could be accomplished through certain commercial systems which utilize flexible membranes on which cells are grown and stretched. Potentially, one could also co-culture iPSC-derived endocardial cells with contractile cardiomyocytes on a deformable hydrogel platform, recreating not only the mechanical contraction, but also the in vivo signaling axis between cardiomyocytes and endocardial cells.

\section{Conclusion \& future perspective}

In light of the shortcomings of previously tested cell types for TEHVs, it is clear that a novel approach to generate engineered VICs is needed, and iPSCs could be used to meet this need. Traditional methods of directing iPSC differentiation will likely be insufficient to recreate the unique valve microenvironment and will necessarily require modification to account for the mechanical environment of the developing valve. Once established, such techniques could lead to the generation of patient-derived VICs that, when used with TEHVs will be less prone to maladaptive remodeling, reduce immune response and have the potential for long-term growth, thereby increasing the efficacy of tissue engineered valve implants.

Financial \& competing interests disclosure

The authors are supported by the NIH (T32-HL007411, R01-HL115103 and R01-HL128715) and Fondation Leducq. The authors have no other relevant affiliations or financial involvement with any organization or entity with a financial interest in or financial conflict with the subject matter or materials discussed in the manuscript apart from those disclosed.

No writing assistance was utilized in the production of this manuscript.

\section{References}

1 Hutcheson JD, Aikawa E, Merryman WD. Potential drug targets for calcific aortic valve disease. Nat. Rev. Cardiol. 11(4), 218-231 (2014).

2 Zilla P, Brink J, Human P, Bezuidenhout D. Prosthetic heart valves: catering for the few. Biomaterials 29(4), 385-406 (2008).

3 Hoffmann G, Lutter G, Cremer J. Durability of bioprosthetic cardiac valves. Dtsch. Arztebl. Int. 105(8), 143-148 (2008).
4 Pibarot P, Dumesnil JG. Prosthetic heart valves. Circulation 119(7), 1034-1048 (2009).

5 Webb JG, Wood DA. Current status of transcatheter aortic valve replacement. J. Am. Coll. Cardiol. 60(6), 483-492 (2012).

6 Sewell-Loftin MK, Chun YW, Khademhosseini A, Merryman WD. EMT-inducing biomaterials for heart valve engineering: taking cues from developmental biology. J. Cardiovasc. Transl. Res. 4(5), 658-671 (2011)
7 Jana S, Tranquillo RT, Lerman A. Cells for tissue engineering of cardiac valves. J. Tissue Eng. Regen. Med. 10(10), 804-824 (2016).

8 Cheung DY, Duan B, Butcher JT. Current progress in tissue engineering of heart valves: multiscale problems, multiscale solutions. Expert Opin. Biol. Ther. 15(8), 1155-1172 (2015).

9 Takahashi K, Yamanaka S. Induction of pluripotent stem cells from mouse embryonic and adult fibroblast cultures by defined factors. Cell 126(4), 663-676 (2006). 
10 Cao J, Li X, Lu X, Zhang C, Yu H, Zhao T. Cells derived from iPSC can be immunogenic - yes or no? Protein Cell 5(1), 1-3 (2014).

11 Rufaihah AJ, Huang NF, Kim J et al. Human induced pluripotent stem cell-derived endothelial cells exhibit functional heterogeneity. Am. J. Transl. Res. 5(1), 21-35 (2013).

12 Granados-Riveron JT, Brook JD. The impact of mechanical forces in heart morphogenesis. Circ. Cardiovasc. Genet. 5(1), 132-142 (2012).

13 Menon V, Eberth JF, Goodwin RL, Potts JD. Altered hemodynamics in the embryonic heart affects outflow valve development. J. Cardiovasc. Dev. Dis. 2(2), 108-124 (2015).

14 Ten Dijke P, Egorova AD, Goumans MJ, Poelmann RE, Hierck BP. TGF- $\beta$ signaling in endothelial-to-mesenchymal transition: the role of shear stress and primary cilia. Sci. Signal. 5(212), 2 (2012).

15 Mahler GJ, Frendl CM, Cao Q, Butcher JT. Effects of shear stress pattern and magnitude on mesenchymal transformation and invasion of aortic valve endothelial cells. Biotechnol. Bioeng. 111(11), 2326-2337 (2014).

16 Yalcin HC, Shekhar A, McQuinn TC, Butcher JT. Hemodynamic patterning of the avian atrioventricular valve. Dev. Dyn. 240 (1), 23-35 (2011).

17 Schroer AK, Merryman WD. Mechanobiology of myofibroblast adhesion in fibrotic cardiac disease. J. Cell Sci. 128(10), 1865-1875 (2015).

18 Sewell-Loftin MK, DeLaughter DM, Peacock JR et al. Myocardial contraction and hyaluronic acid mechanotransduction in epithelial-to-mesenchymal transformation of endocardial cells. Biomaterials 35(9), 2809-2815 (2014).

19 Gould RA, Yalcin HC, MacKay JL et al. Cyclic mechanical loading is essential for Rac1-mediated elongation and remodeling of the embryonic mitral valve cyclic mechanical loading is essential for Rac1-mediated elongation and remodeling of the embryonic mitral valve. Curr. Biol. 26(1), 27-37 (2016)

20 Ruan JL, Tulloch NL, Razumova MV et al. Mechanical stress conditioning and electrical stimulation promote contractility and force maturation of induced pluripotent stem cell-derived human cardiac tissue. Circulation 134(20), 1557-1567 (2016). 